\title{
El tratamiento con implantes cigomáticos en pacientes con atrofia maxilar severa
}

\section{Treatment with zygomatic implants in patients with advanced atrophy of maxilla}

O. Guerra Cobián*, I. Grau León**, A. Jiménez Guerra***, I. Ortiz García***, B. Ramos Medina****, C. Sánchez Silot*****

\section{RESUMEN}

Introducción. El objetivo del presente estudio era mostrar los resultados del tratamiento con implantes cigomáticos utilizados en pacientes con atrofia maxilar severa.

Metodos. 18 pacientes fueron tratados con 36 implantes cigomáticos Galimplant @ sumergidos con conexión interna para su rehabilitación prostodóncica. Las técnicas quirúrgicas de inserción de cigomáticos fueron extrasinusal y la técnica de la ranura sinusal. Los implantes fueron cargados después de un periodo de cicatrización de 6 meses.

Resultados. Los hallazgos clínicos indican una supervivencia de los implantes del 100\%. 14 implantes (38,9\%) se insertaron por via extrasinusal y 22 implantes $(61,6 \%)$ por la técnica de la ranura sinusal. Un total de 18 prótesis híbridas atornilladas fueron realizadas. Complicaciones postoperatorias fueron observadas en 4 pacientes (22,2\%). El periodo medio de seguimiento clínico ha sido de 12 meses.

Conclusiones. Este estudio indica que los implantes cigomáticos pueden ser utilizados con éxito en el tratamiento de los pacientes con atrofia maxilar severa.

PALABRAS CLAVE: Implantes dentales, implantes cigomáticos, atrofia maxilar, complicaciones implantológicas, prótesis sobre implantes, implantología oral.

\section{ABSTRACT}

Introduction. The aim of this study was to report the outcome of treatment with zygomatic implants used in patients with severe atrophy of maxilla.

Methods. 18 patients were treated with 36 Galimplant ${ }^{\circledR}$ zygomatic implants with internal connection for prosthodontic rehabilitation. Zygomatic surgical techniques were two approach for insertion of implants, extrasinusal and sinus slot technique. Implants were loaded after a healing free-loading period of six months. Results. Clinical results indicate a survival rate of implants of $100 \%$. Fourteen implants (38.9\%) were inserted by extrasinusal approach while twenty two implants were inserted by sinus slot approach (61.1\%). A

* Profesor de Cirugía Oral e Implantología. Especialista en Cirugía Máxilofacial. Facultad de Estomatología. Universidad de Ciencias Médicas. La Habana.

** Profesora Titular. Doctora en Ciencias Estomatológicas. Especialista en Prótesis Estomatológica y Estomatología General Integral. Facultad de Estomatología. Universidad de Ciencias Médicas. La Habana.

*** Profesor Asociado de Odontología Integrada de Adultos y de Gerodontología. Profesor del Máster de Implantologia Oral. Facultad de Odontología. Universidad de Sevilla.

**** Jefe del Servicio de Cirugía Oral y Máxilofacial. Hospital Universitario Santa Lucía de Cartagena. Murcia.

***** Profesora de Prótesis Estomatológica. Doctora en Ciencias Estomatológicas. Facultad de Estomatología. Universidad de Ciencias Médicas. La Habana. 
total of 18 hybrid screw prostheses were realized. Postoperative complications were observed in four patients (22.2\%). The mean follow-up period were 12 months.

Conclusions. This study indicate that zygomatic implants can be used with success in treatment of patients with severe atrophic maxilla.

KEY WORDS: Dental implants, zygomatic implants, atrophic maxilla, implant complications, implant-supported prosthesis, implant dentistry.

Fecha de recepción: 23 de marzo de 2020

Fecha de aceptación: 30 de marzo de 2020

O. Guerra Cobián, I. Grau León, A. Jiménez Guerra, I. Ortiz García, B. Ramos Medina, C. Sánchez Silot. El tratamiento con implantes cigomáticos en pacientes con atrofia maxilar severa. 2020; 36, (2): 71-79.

\section{INTRODUCCIÓN}

Desde un punto de vista, sobre todo quirúrgico, el maxilar superior supone un reto importante para el tratamiento con implantes por su anatomía y por la frecuente escasa disponibilidad y calidad de hueso debido a la pérdida prematura de su dentición natural ${ }^{1-4}$. La atrofia severa del maxilar superior exige la realización de técnicas quirúrgicas complejas para la colocación de los implantes como son los injertos óseos autólogos. En este sentido, se ha descrito también la elevación del seno maxilar, la inclinación de los implantes aprovechando los arbotantes que separa el seno maxilar de la fosa nasal y la colocación de implantes con fijación en la apófisis pterigoides y en el cuerpo del hueso cigomático. En los segmentos posteriores, se prioriza la necesidad de insertar implantes lo suficientemente largos que permitan soportar adecuadamente las fuerzas masticatorias $^{1-4}$.

Los implantes cigomáticos se han convertido en una opción eficaz en el tratamiento de los pacientes con atrofia severa del maxilar superior. Los implantes cigomáticos representan una buena solución para conseguir una fijación maxilar posterior y poder rehabilitar al paciente, casi siempre de forma inmediata ${ }^{5-6}$. La inserción de los implantes cigomáticos es una técnica quirúrgica compleja que se realiza sin injertos óseos y que incorpora implantes de una longitud aproximada de 35-50 $\mathrm{mm}$ en el hueso malar o cigoma, emergiendo sus conexiones a nivel premolar en el paladar para su posterior rehabilitación prostodóncica ${ }^{7-8}$.

El tratamiento del paciente con implantes cigo- máticos requiere un plan de tratamiento complejo que incluye el diagnóstico clínico y radiológico tomográfico computarizado, un estricto protocolo quirúrgico, una adecuada rehabilitación prostodóncica y para conseguir el éxito a largo plazo un programa de mantenimiento y revisiones periódicas $^{9-11}$.

La técnica original de Branemark preconizaba la colocación bilateral de dos implantes cigomáticos (uno en cada lado) y de 4 implantes convencionales en el sector anterior maxilar ${ }^{12}$. Los implantes cigomáticos se colocaban a través del seno maxilar y emergían por la cara palatina de la cresta alveolar. Posteriormente, este protocolo fué modificado por la colocación de 4 implantes cigomáticos, dos en cada lado, sobre todo en los pacientes con insuficiente volumen óseo en el sector anterior maxilar ${ }^{13}$.

En los últimos 20 años, se han modificado sustancialmente los procedimientos quirúrgicos de inserción de los implantes cigomáticos ${ }^{7,11,14-15}$. Además de la via clásica intrasinusal, se ha propuesto la via extrasinusal donde el punto de entrada del implante se realiza mediante una osteotomía en la cresta alveolar y la colocación se realiza externamente a la membrana sinusal ${ }^{14,16}$. Se ha descrito también, una vía intermedia sobre la pared externa del seno maxilar mediante la creación de un trayecto o ranura sinusal que va desde aproximadamente el suelo del seno maxilar hasta la base del cigoma ${ }^{14,17}$.

La incidencia de complicaciones como mucositis/ periimplantitis en las uniones implante-pilar, sinusitis, alteraciones fonéticas y diseños prostodón- 
cicos demasiados extensos han desarrollado una nueva filosofia de tratamiento con implantes cigomáticos basada en el concepto de cirugía guiada por la anatomía del paciente ${ }^{5,11}$. En este sentido, la trayectoria del implante cigomático puede ser intra o extrasinusal con respecto al seno maxilar para lograr que la cabeza del implante tenga su punto de emergencia en la cresta alveolar y permita una rehabilitación prostodóncica más favorable del paciente ${ }^{5,11}$.

El objetivo del presente estudio era evaluar el tratamiento con implantes cigomáticos en la rehabilitación de los pacientes con un maxilar superior atrófico.

\section{PACIENTES Y METODOS.}

Se realiza una investigación relacionada con el tratamiento de implantes cigomáticos en los pacientes atendidos en la Facultad de Estomatología de la Universidad de Ciencias Médicas de La Habana en el Programa de Implantología Oral que se desarrolla para la atención de los pacientes con diversos grados de edentulismo parcial o total.

Todos los pacientes seleccionados en el estudio fueron evaluados, clínicamente con un examen oral completo y una valoración prostodóncica de ambos maxilares. Radiológicamente, en todos los pacientes se realizaron una ortopantomografía y posteriormente, una tomografía computarizada.

FASE QUIRURGICA. Los pacientes fueron intervenidos para la inserción quirúrgica de los implantes cigomáticos mediante un protocolo básico de cirugía de colgajo que se correspondía con la realización del fresado sin otro tipo de intervención.

Todos los pacientes recibieron anestesia loco-regional. Una hora antes de la cirugía, los pacientes comenzaron un régimen antibiótico con amoxicilina durante una semana. En casos de molestias, dolor o inflamación a todos los pacientes se les recomendó ibuprofeno.

La cirugía de inserción de los implantes cigomáticos consistió en la realización de una incisión a nivel crestal del reborde alveolar edéntulo maxilar y un despegamiento de espesor total para exponer el hueso maxilar y el hueso cigomático. Se realizó una técnica quirúrgica yuxtasinusal mediante el fresado de la pared externa maxilar y la creación

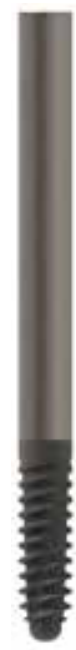

Figura 1. Imagen de los implantes cigomáticos IPX Smooth

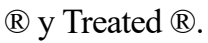

de un lecho o ranura sinusal. En los casos menos favorables se realizó una técnica extrasinusal. Los extremos apicales de los implantes fueron insertados en el cigoma y obtuvieron una buena estabilidad primaria. No se utilizaron injertos ni biomateriales. Posteriormente se realizó la sutura del colgajo.

En la cirugía se utilizaron implantes cigomáticos IPX® de conexión interna y superficie arenada y grabada Nanoblast @, fabricados por Galimplant (Sarria, España), registrados por el Centro de Control Estatal de Equipos Médicos (CCEEM) del Ministerio de Salud Pública (MINSAP) de la República de Cuba. Se utilizaron dos tipos de estos implantes cigomáticos, IPX Smooth $®$ con superficie tratada solamente en su extremo apical para la técnica extrasinusal e IPX Treated $₫$ con superficie totalmente tratada para la técnica de la ranura sinusal (Figura 1)

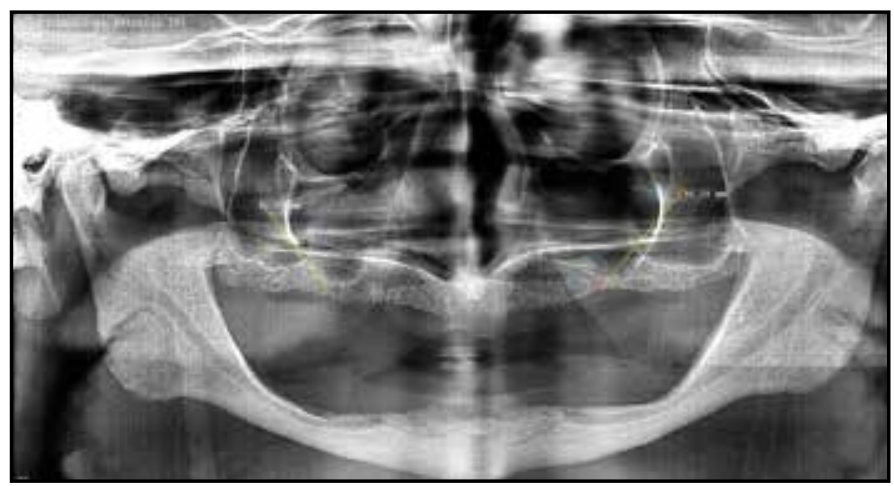

Figura 2. Ortopantomografía previa de un paciente edéntulo total.

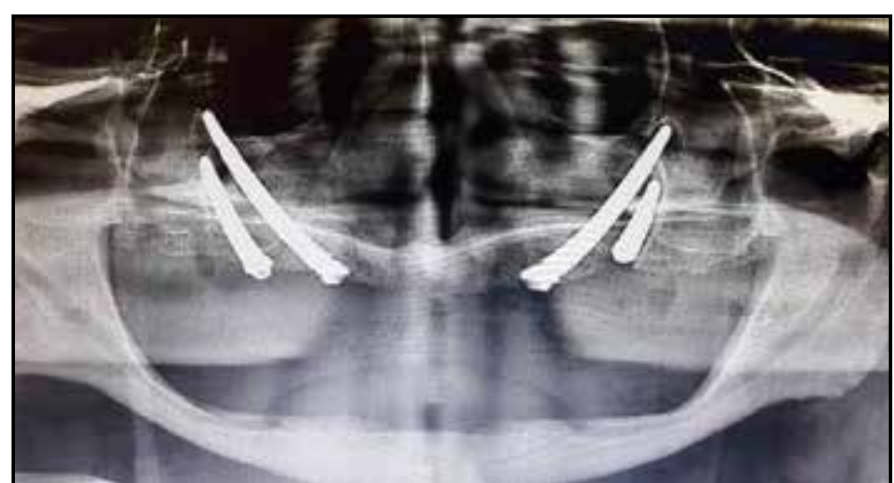

Figura 3. Ortopantomografía de un paciente edéntulo total con los implantes cigomáticos insertados. 
Los pacientes intervenidos fueron evaluados clínica y radiológicamente después de la cirugía, durante la realización de la técnica prostodóncica y posteriormente durante la fase de mantenimiento del tratamiento (Figuras 2 y 3 ).

REHABILITACION PROTESICA. A los 6 meses, después de la inserción de los implantes se realizó la fase prostodóncica, mediante la carga funcional de los implantes cigomáticos. Previamente, se realizó un examen clínico y un control radiológico con una ortopantomografía para observar la situación de los implantes. Se utilizaron pilares transepiteliales para compensar las angulaciones de los implantes. Dos semanas después se tomaron las correspondientes impresiones para la confección de las prótesis híbridas implantosoportadas de metal-resina.

El tiempo transcurrido de seguimiento clínico desde la carga funcional de los implantes ha sido de 12 meses.

CONSIDERACIONES ETICAS. Mediante el consentimiento informado, los pacientes fueron informados por los investigadores del protocolo diagnóstico, del tratamiento quirúrgico, rehabilitacion protésica y mantenimiento. También conocieron los beneficios esperados por el tratamiento, posibles complicaciones y los cuidados de que iban a ser objeto. Se aseguró del acceso a la adecuada alternativa de tratamiento rehabilitador en caso de fracasar la terapéutica realizada.

ANALISIS ESTADISTICO. La fuente de información que se empleó fue la historia clínica de cada paciente, los datos se recolectaron mediante un modelo confeccionado al efecto, con lo que se creó una base de datos automatizada en SPSS versión 15.0 para Windows que permitió realizar los análisis estadísticos descriptivos con referencia a las variables demográficas de los pacientes, los implantes cigomáticos, las prótesis realizadas y las complicaciones.

\section{RESULTADOS}

PACIENTES. 18 pacientes (11 hombres y 7 mujeres) fueron tratados con implantes cigomáticos, con una edad media de 54,2 años (rango: 3671 años). 2 pacientes $(11,1 \%)$ eran fumadores. 3 pacientes $(16,7 \%)$ presentaban alguna patología

\begin{tabular}{lcc}
\multicolumn{3}{c}{ Tabla 1. Distribucion de los pacientes } \\
Edad: 54,2 años & $\mathbf{N}^{\circ}$ & $\%$ \\
Sexo & 11 & $61,1 \%$ \\
Hombres & 7 & $38,9 \%$ \\
Mujeres & 2 & $11,1 \%$ \\
Fumadores & 3 & $16,7 \%$ \\
Otras Patologías & 4 & $22,2 \%$ \\
Complicaciones & \\
\hline
\end{tabular}

\begin{tabular}{lcc}
\multicolumn{3}{c}{ Tabla 2. Distribucion de los implantes } \\
Número & $\mathbf{N}^{\circ}$ & $\%$ \\
Tipo & 36 & $100 \%$ \\
Smooth & & \\
Treated & 14 & $38,9 \%$ \\
Diámetro & 22 & $61,1 \%$ \\
$4,2 \mathrm{~mm}$ & & \\
$4,3 \mathrm{~mm}$ & 14 & $38,9 \%$ \\
Longitud & 22 & $61,1 \%$ \\
$35 \mathrm{~mm}$ & & \\
$37,5 \mathrm{~mm}$ & 6 & $16,7 \%$ \\
$40 \mathrm{~mm}$ & 4 & $27,8 \%$ \\
$42,5 \mathrm{~mm}$ & 10 & $11,1 \%$ \\
$45 \mathrm{~mm}$ & 3 & $8,3 \%$ \\
$50 \mathrm{~mm}$ & 7 & $19,4 \%$ \\
\hline
\end{tabular}

sistémica (un paciente diabético, un paciente con hipotiroidismo y un paciente con osteoporosis).

CIRUIGIA DE IMPLANTES. Se colocaron un total de 36 implantes cigomáticos en los correspondientes 18 pacientes. 4 implantes cigomáticos en 4 pacientes (16 implantes), 2 implantes bilaterales en 6 pacientes (12 implantes) y 8 implantes unilaterales, 5 en el lado izquierdo y 3 en el lado derecho.

14 implantes $(38,9 \%)$ IPX Smooth @ (solamente tratados en el extremo apical) fueron insertados mediante la técnica extrasinusal y 22 implantes $(61,1 \%)$ IPX Treated @ (totalmente tratados con arenado y grabado en su superficie) fueron insertados mediante la técnica de la ranusal sinusal.

Con respecto al diámetro, 22 implantes cigomáticos $(61,1 \%)$ eran de $4,3 \mathrm{~mm}$, y 14 implantes $(38,9 \%)$ eran de $4,2 \mathrm{~mm}$. Con respecto a la longitud, 4 implantes $(11,1 \%)$ eran de $35 \mathrm{~mm}, 6$ implantes $(16,7 \%)$ eran de $37,5 \mathrm{~mm}, 10$ implantes (27,8\%) eran de $40 \mathrm{~mm}, 3$ implantes (8,3\%) eran de $42,5 \mathrm{~mm}, 7$ implantes $(19,4 \%)$ eran de $45 \mathrm{~mm}$ y 6 implantes $(16,7 \%)$ eran de $50 \mathrm{~mm}$.

En la investigación realizada no se perdieron implantes. Se observaron complicaciones inmediatas postquirúrgicas en 4 pacientes (22,2\%), dos casos de edema en la zona intervenida, un caso 
de equimosis facial y un caso de parestesia infraorbitaria transitoria.

REHABILITACIÓN PROTESICA. Se realizaron un total de 18 prótesis híbridas atornilladas de metal-resina.

\section{DISCUSIÓN}

Los resultados de este estudio demuestran que los implantes cigomáticos pueden ser insertados con éxito y obtener una oseointegración adecuada para su carga funcional prostodóncica. Este trabajo presenta los datos de la inserción de implantes cigomáticos en pacientes parcial y totalmente edéntulos, demostrando una supervivencia de los implantes del $100 \%$ después de un periodo de seguimiento clínico de 1 año.

La mayoría de los pacientes de esta investigación no presentaban enfermedades sistémicas, aunque algunos pacientes padecían diabetes mellitus, hipotiroidismo y osteoporosis (16,7\%). 2 pacientes $(11,1 \%)$ eran fumadores. En este sentido, se han aplicado en estudios con implantes cigomáticos como criterios de exclusión la presencia de patología médicas como enfermedades hematológicas, coagulopatías, diabetes descontrolada, radioterapia en la región maxilofacial, trastornos psíquicos o consumo de más de 20 cigarrillos diarios ${ }^{7,18}$.

Un aspecto importante de la cirugía de implantes cigomáticos es la anestesia de los pacientes. La anestesia puede ser loco-regional, loco-regional con sedación y la anestesia general. En la presente investigación la anestesia fué loco-regional. En los casos de sedación intravenosa y anestesia general, los pacientes deben ser evaluados de sus funciones vitales previamente por el anestesista para conocer su estado sistémico y farmacológico para evitar accidentes durante la intervención ${ }^{7,19}$.

Todos los pacientes del estudio fueron medicados con antibióticos durante una semana con amoxicilina. Este protocolo se utilizó como prevención de posibles complicaciones infecciosas. Además de la administración de antibióticos, todos los pacientes utilizaron un colutorio de clorhexidina después de la cirugía implantológica, durante los 30 días posteriores, con el mismo fin de prevención de la bacteriemia postquirúrgica. En este sentido, la prescripción de antibióticos y antiinflamatorios suele ser el protocolo farmacológico habitual en la cirugía de implantes cigomáticos ${ }^{10,20}$.

El diagnóstico del paciente para implantes cigomáticos requiere la valoración de la estructura ósea mediante el examen radiológico ${ }^{15,19}$. En esta investigación en relación a la selección de los pacientes, en una primera fase se realizó una ortopantomografía como medio diagnóstico por imagen para valorar el grado de disponibilidad ósea. Posteriormente, en los casos seleccionados para la cirugía de implantes cigomáticos se realizaron tomografías computarizadas. Este doble examen radiológico es observado frecuentemente en los estudios publicados ${ }^{7,15,19}$. Para complementar el diagnóstico radiológico, los investigadores realizaron una exploración clínica minuciosa con especial atención en la inspección y palpación de los rebordes alveolares residuales.

La técnica quirúrgica clásica o convencional de implantes cigomáticos fué desarrollada en 1988 según el protocolo original de Branemark y cols. 12. En este protocolo quirúrgico original con implantes cigomáticos se utilizaban implantes con hexágono externo y superficie mecanizada e insertados de forma intrasinusal. Se realiza una ventana en la pared lateral del seno y se despega la membrana de Schneider para realizar la secuencia de fresado. Con esta técnica se ha obtenido una tasa de éxito muy elevada ${ }^{12}$. Posteriormente este protocolo fué aplicado a nivel internacional por investigadores de 16 centros que trataron a 75 pacientes con 420 implantes incluyendo 145 implantes cigomáticos con un $97,9 \%$ de supervivencia a 1 año ${ }^{21}$. A los 3 años, el $75 \%$ de las localizaciones de implantes presentaban una mucosa periimplantaria normal y un $68 \%$ sin placa ${ }^{22}$. La tasa de supervicencia fué del $96,3 \%$. Los pacientes estaban satisfechos con la función (71\%) y la estética (86\%) de la rehabilitación ${ }^{22}$.

En la presente investigación, 18 pacientes fueron tratados con la técnica quirúrgica yuxta o extrasinusal de cirugía con implantes cigomáticos mediante la apertura del colgajo de espesor total, la exposición del hueso maxilar y el hueso malar, el fresado de los lechos implantarios, la inserción de los implantes y la sutura correspondiente. Desde un punto de vista quirúrgico, para la inserción de la mayoría de los implantes $(61,1 \%)$ se realizó la técnica quirúrgica yuxtasinusal o de la ranura sinusal, mientras que el $38,9 \%$ de los implantes fue- 
ron insertados mediante la técnica extrasinusal.

La técnica yuxtasinusal o de la ranura sinusal (sinus slot technique, en ingles) fué introducida por Stella y Warner ${ }^{17}$ representando una alternativa a la técnica original de Branemark. Se realiza una pequeña antrostomía lateral del seno que va ser utilizada posteriormente como orientación de las fresas para crear un trayecto óseo para la colocación del implante cigomático. En este sentido, esta técnica posibilita un mejor control de la posición tridimensional del implante y minimiza su presencia a través del seno maxilar. Además, al colocar el implante cigomático más axial, la emergencia de la parte coronal es más vestibular y favorece una rehabilitación prostodóncica menos palatinizada, más favorable para el paciente ${ }^{14-15}$. Los resultados clínicos de la técnica yuxtasinusal o de la ranura sinusal en la cirugía cigomática son muy favorables, con una tasa de superviencia del $100 \%$ a los 18 meses, aunque son necesarios más estudios a largo plazo ${ }^{23}$.

La técnica extrasinusal para la cirugía de implantes cigomáticos se realiza en aquellos casos de la presencia de una pronunciada concavidad vestibular en el maxilar que obligaba a colocar la parte coronal del implante muy palatinizada lejos de la cresta alveolar ${ }^{14,24}$. Esta técnica permite visualizar directamente el implante cigomático ya que es colocado externamente al hueso maxilar cubierto por los tejidos blandos, siendo el cigoma su única fijación. Esta técnica quirúrgica es menos invasiva y reduce el tiempo de intervención y las molestias postoperatorias ${ }^{14,24}$. Los resultados clínicos de la técnica extrasinusal en la cirugía de implantes cigomáticos son muy favorables, las tasas de supervivencia se sitúan en el 98-100\% 16,18,24-25.

En 2011, Aparicio y cols ${ }^{26}$ presentaron una clasificación anatómica con 5 tipos de cirugía cigomática basada en la posición de los implantes cigomáticos colocados obtenida de los resultados de 200 localizaciones en 100 pacientes según los principios quirúrgicos y prostodóncicos de un enfoque de cigomáticos guiado por la anatomía (ZAGA, zygoma anatomy guided approach, en inglés). En 2013, este grupo de investigadores presentaron los resultados de una cohorte de 80 pacientes consecutivos tratados con implantes cigomáticos según la técnica ZAGA 27. El estudio tuvo un seguimiento de al menos 3 años. 442 implantes convencionales fueron colocados y 157 implantes cigomáticos. Se perdieron 4 implantes cigomáticos y 20 implantes convencionales. La tasa de supervivencia fue del $95,5 \%{ }^{27}$. Esta técnica ZAGA se ha ido consolidando y aportando mayor experiencia clínica ya que ha impulsado una nueva filosofía de cirugía con implantes cigomáticos que pueden ser intra, yuxta o extrasinusales pero siempre basada en las características anatómicas del pacientes obtenidas por su examen clínico y tomográfico computarizado, es decir por su diagnóstico individualizado ${ }^{5,7,14,26-27}$. Este enfoque ZAGA proporciona a los clínicos una guía para conseguir una posición ideal de los implantes con fines prostodóncicos, una menor invasión sinusal y una estabilidad de los tejidos duros periimplan$\operatorname{tarios}^{5,7,14,26-27}$.

En la presente investigación, se colocaron 36 implantes cigomáticos en los 18 pacientes con una tasa de supervivencia después de 1 año de seguimiento clínico del 100\%. En general, las tasas de supervivencia de los implantes cigomáticos es muy elevada. Las diferentes técnicas intra y extrasinusales ofrecen resultados muy favorables por encima del $90 \%$ de éxito del tratamiento 12,14-15,22,2427. Un estudio a 18 años presenta los hallazgos de 88 implantes cigomáticos en 45 pacientes. 54 implantes $(61,3 \%)$ fueron cargados de forma inmediata, siendo la tasa de supervivencia del 94,3\% 28. En este sentido, una revisión sistemática sobre implantes cigomáticos realizada sobre 68 estudios con 4556 implantes colocados en 2161 pacientes refiere 103 fracasos de implantes a lo largo de un periodo de 12 años, lo que representa una tasa de supervivencia del 95,2\% ${ }^{28}$. La mayoría de los fracasos de implantes tuvieron lugar en el periodo de 6 meses después de la carga funcional. Los estudios con protocolos de carga inmediata presentaron menos fracasos que los estudios con carga diferida ${ }^{29}$.

En la presente investigación, se observaron complicaciones inmediatas postquirúrgicas en 4 pacientes $(22,2 \%)$, dos casos de edema en la zona intervenida, un caso de equimosis facial y un caso de parestesia infraorbitaria transitoria. No se observaron sinusitis. Las complicaciones en el tratamiento con implantes cigomáticos son relativamente frecuentes ${ }^{18,20,30}$. Un estudio a 6 años con cirugía extrasinusal de implantes cigomáticos presenta perforaciones de la membrana de Schneider en el $10 \%$ de las cirugías y un caso de comunicación oroantral ${ }^{18}$. La incidencia de si- 
nusitis en los implantes cigomáticos colocados de forma extrasinusal suel ser mínima o ausente 16. La incidencia de rinosinusitis es valorada en un estudio a 35 meses de seguimiento donde la técnica intrasinusal fué la más utilizada $(43,8 \%)$ 30 . Los resultados sugieren que las alteraciones en los senos paranasales en los pacientes intervenidos pueden ser consecuencia en la mayoría de las veces de patología subclínica previa diagnosticada por tomografía computarizada en el preoperatorio ${ }^{30}$. Un estudio a 4 años con implantes cigomáticos intrasinusales presenta como complicaciones la presencia de infección local y de sinusitis en el $56,2 \%$ y el $37,5 \%$ de los pacientes, respectivamente ${ }^{20}$. La revisión de la literatura ha mostrado que las complicaciones más frecuentes son la sinusitis $(2,4 \%)$, la infección de los tejidos blandos (2\%), la parestesia (1\%) y las fistulas oroantrales $(0,4 \%)^{29}$. Aunque estas prevalencias pueden ser subestimadas porque muchos estudios no mencionan la frecuencia de las complicaciones. La complicación más importante es la sinusitis que puede aparecer incluso años después de la cirugía ${ }^{29}$.

El objetivo final de la cirugía con implantes cigomáticos es la rehabilitación de los pacientes edéntulos maxilares con su prótesis correspondiente implantosoportada. En la presente investigación, la carga funcional fué diferida, después de 6 meses de la colocación de los implantes cigomáticos. Los diversos estudios de tratamiento con implantes cigomáticos presentan protocolos de carga funcional diferida, precoz e inmediata, siendo la prótesis híbrida atornillada inmediata la más utilizada ${ }^{7,10,20,25}$. Desde un punto de vista biomecánico, los implantes cigomáticos por su longitud y por su reducida superficie de unión al cigoma, son afectados sobre todo por las fuerzas oclusales horizontales o transversales. En este sentido, la rehabilitación prostodóncica debe asegurar una rígida conexión de los implantes a las prótesis. Además, en todos los casos necesarios, los implantes cigomáticos deben ser conectados por la prótesis a otros implantes convencionales que se hayan insertados para el tratamiento global del maxilar superior edéntulo ${ }^{24}$.

La carga inmediata ha representado el protocolo clínico más frecuente para la rehabilitación de los pacientes intervenidos con implantes cigomáticos $7,10,20,25$. Una revisión sistemática refleja los resultados de 38 estudios con un seguimiento medio de 12 meses, de los resultados clínicos de la carga inmediata ${ }^{6}$. Las conclusiones de esta revisión subrayan que los implantes cigomáticos cargados inmediatamente después de la cirugía representan una alternativa viable para la rehabilitación de los pacientes con atrofia maxilar severa ${ }^{6}$.

Un estudio reciente presenta los resultados de rehabilitaciones fijas soportadas con implantes cigomáticos cargados de forma inmediata (35 pacientes) comparada con implantes convencionales en maxilares regenerados con xenoinjertos cargados a los 4 meses (36 pacientes). El seguimiento clínico fué de 4 meses después de la carga funcional ${ }^{31}$. Los pacientes tratados con implantes cigomáticos presentaron menos fracasos de implantes (un paciente perdió 3 implantes vs 35 implantes en 8 pacientes) y de prótesis ( 1 vs 6 pacientes) comparados con los pacientes tratados con implantes convencionales ${ }^{31}$.

Un aspecto importante del tratamiento con implantes cigomáticos para la rehabilitación del maxilar atrófico es el grado de satisfacción de los pacientes con esta opción terapeútica ${ }^{32}$. Es necesario considerar que aunque este tipo de tratamiento quirúrgico es complejo y muy invasivo, ya que comprende generalmente anestesia general o sedación intravenosa, sin embargo el número y duración de la fase quirúrgica es menor que la técnica convencional con injertos de hueso autólogo. Además, cada vez se ha mejorado más con las nuevas técnicas y materiales, el diseño de las prótesis que se han optimizado en función y en estética. En general, los pacientes presentan un elevado nivel de satisfacción que está también relacionado con el éxito muy favorable del tratamiento ${ }^{32}$.

\section{CONCLUSIONES}

La rehabilitación prostodóncica de pacientes con atrofia severa del maxilar superior mediante la inserción de implantes cigomáticos representa un tratamiento con un éxito elevado en los pacientes que han perdido sus dientes naturales. La realización de un protocolo estricto de cirugía implantológica basado en el diagnóstico clínico y radiológico permite la inserción de los implantes cigomáticos de forma segura y predecible. La carga funcional con las prótesis correspondientes sobre los implantes permite re- 
habilitar funcional y estéticamente al paciente y mejorar su calidad de vida.

\section{BIBLIOGRAFIA}

1. Barone A, Varanini P, Orlando B, Tonelli P, Covani U. Deep-frozen allogenic onlay bone grafts for reconstruction of atrophic maxillary alveolar ridges: a preliminary study. J Oral Maxillofac Surg 2009; 67: 1300-1306.

2. Chiapasco M, Romeo E, Coggiola A, Brusati R. Long-term outcome of dental implants placed in revascularized fibula free flaps used for the reconstruction of maxillo-mandibular defects due to extreme atrophy. Clin Oral Implants Res 2011; 22: 83-91.

3. Baj A, Trapella G, Lauritano D, Candotto V, Mancini GE, Gianni AB. An overview on bone reconstruction of atrophic maxilla: success parameters and critical issues. J Biol Regul Homeost Agents 2016; 30:209-215.

4. Lorrio Castro JM, Sierra Armas L, Lorrio Castro C, García Ávila I, Monsalve Guil L, Gómez Font R. Cirugía compleja en el tratamiento con implantes del maxilar superior. Av Odontoestomatol 2017; 33:213-220.

5. Aparicio C, Manresa C, Francisco K, Claros P, Alández J, González-Martín O, Albrektsson T. Zygomatic implants: indications, techniques and outcomes, and the zygomatic success code. Periodontol 2000 2014; $66: 41-58$

6. Tuminelli FJ, Walter LR, Neugarten J, Bedrossian E. Immediate loading of zygomatic implants: A systematic review of implant survival, prosthesis survival and potential complications. Eur J Oral Implantol 2017; 10 Suppl 1:79-87.

7. Davó R, Bankauskas S, Laurincikas R, Koçyigit ID, Maté-Sánchez de Val JE. Clinical performance of zygomatic implants-retrospective multicenter study. J Clin Med 2020; 9: 480.

8. Rosenstein J, Dym H. Zygomatic implants: A solution for the atrophic maxilla. Dent Clin North Am 2020; 64:401-409.
9. Aparicio C, López-Piriz R, Albrektsson T. ORIS criteria of success for the zygoma-related rehabilitation: The (revisited) zygoma success code. Int J Oral Maxillofac Implants 2020; 35: 366-378.

10. Balan I, di Girolamo M, Lauritano D, Carinci F. Treatment of severe atrophic maxilla with zygomatic implants: a case series. Oral Implantol 2017; 10:317-324.

11. Almeida PHT, Cacciacane SH, Arcazas Jr A. Ten-year follow-up of treatment with zygomatic implants and replacement of hybrid dental prosthesis by ceramic teeth: A case report. Ann Med Surg 2020; 50: 1-5.

12. Brånemark P-I, Gröndahl K, Öhrnell L-O, NiIsson P, Petruson B, Svensson B, Engstrand $P$, Nannmark U. Zygoma fixture in the management of advanced atrophy of the maxilla: technique and longterm results. Scand J Plast Reconstr Surg Hand Surg 2004; 38:70-85.

13. Bothur S, Jonsson G, Sandahl L. Modified technique using multiple zygomatic implants in reconstruction of the atrophic maxilla: a technicalnote. Int J Oral Maxillofac Implants. 2003;18:902-904.

14. Sharma A, Rahul GR. Zygomatic implants/ fixture: a systematic review. J Oral Implantol 2013; 39: 215-234.

15. Grecchi F, Bianchi AE, Siervo S, Grecchi E, Lauritano D, Carinci F. A new surgical and technical approach in zygomatic implantology. Oral Implantol 2017; 10:197-208.

16. Migliorança RM, Coppedê A, Dias Rezende RC, de Mayo T. Restoration of the edentulous maxilla using extrasinus zygomatic implants combined with anterior conventional implants: a retrospective study. Int $\mathrm{J}$ Oral Maxillofac Implants 2011; 26: 665-672.

17. Stella JP, Warner MR. Sinus slot technique for simplification and improved orientation of zygomaticus dental implants: a technical note. Int $\mathrm{J}$ Oral Maxillofac Implants 2000;15:889-893.

18. Agliardi EL, Romeo D, Panigatti S, Nobre 
SMA, Maló P. Immediate full-arch rehabilitation of the severely atrophic maxilla supported by zygomatic implants: a prospective clinical study with minimum follow-up of 6 years. Int $\mathrm{J}$ Oral Maxillofac Surg 2017; 46: 1592-1599.

19. Aleksandrowicz P, Kusa-Podkánska M, Tomkiewicz W, Kotuła L, Perek J, Wysokinska-Miszczuk J. Platform switch hybrid zygoma implants improve prosthetics and marginal bone protection after extra-sinus placement. Clin Implant Dent Relat Res 2020;1-7.

20. Becktor JP, Isaksson S, Abrahamsson P, Sennerby L. Evaluation of 31 zygomatic implants and 74 regular dental implants used in 16 patients for prosthetic reconstruction of the atrophic maxilla with cross-arch fixed bridges. Clin Implant Dent Relat Res 2005; 7:159-165.

21. Hirsch J-M, Öhrnell L-O, Andreasson L, Brånemark P-I, Chiapasco M, Gynther G, et al. A clinical evaluation of the zygoma fixture: one year of follow-up at 16 clinics. J Oral Maxillofac Surg 2004; 62(Suppl 2):22-9.

22. Kahnberg KE, Henry PJ, Hirsch JM, Ohrnell LO, Andreasson L, Brånemark PI, et al. Clinical evaluation of the zygoma implant: 3-year follow-up at 16 clinics. J Oral Maxillofac Surg 2007; 65: 2033-8.

23. Peñarrocha $M$, Uribe R, Garcia B, Martí E. Zygomatic implants using the sinus slot technique: clinical report of a patient series. Int $\mathrm{J}$ Oral Maxillofac Implants 2005;20:788-92.

24. Aparicio C, Ouazzani W, Aparicio A, Fortes V, Muela R, Pascual A et al. Extrasinus zygomatic implants: three year experience from a new surgical approach for patients with pronounced buccal concavities in the edentulous maxilla. Clin Implant Dent Relat Res 2010;12:55-61.

25. Maló P, Nobre M de A, Lopes A, Ferro A, Moss $S$. Five-year outcome of a retrospective cohort study on the rehabilitation of completely edentulous atrophic maxillae with immediately loaded zygomatic implants placed extra-maxillary. Eur J Oral Implantol 2014; 7:267-281.
26. Aparicio C. A proposed classification for zygomatic implant patients based on the zygoma anatomy guided approach (ZAGA). A cross-sectional survey. Eur J Oral Implantol 2011;4:269-275.

27. Aparicio C, Manresa C, Francisco K, Aparicio A, Nunes J, Claros P, Potau JM. Zygomatic implants placed using the zygomatic anatomy-guided approach versus the classical technique. A proposed system to report rhino-sinusitis diagnosis. Clin Implant Dent Relat Res 2013; 16: 627-642.

28. Chana H, Smith G, Bansal H, Zahra D. A retrospective cohort study of the survival rate of 88 zygomatic implants placed over an 18-year period. Int J Oral Maxillofac Implants 2019; 34: 461-470.

29. Chrcanovic BR, Albrektsson T, Wennerberg A. Survival and complications of zygomatic implants: an updated systematic review. J Oral Maxillofac Surg 2016; 74:1949-1964.

30. D'Agostino A, Trevisiol L, Favero V, Pessina $M$, Procacci P, MD, Nocini PF. Are zygomatic implants associated with maxillary sinusitis? J Oral Maxillofac Surg 2016; 74:1562-1573.

31. Esposito M, Davó R, Marti-Pages C, Ferrer-Fuertes A, Barausse C, Pistilli R, Ippolito DR, Felice P. Immediately loaded zygomatic implants vs conventional dental implants in augmented atrophic maxillae: 4 months post-loading results from a multicentre randomised controlled trial. Eur J Oral Implantol 2018; 11: 11-28.

32. Sartori EM, Padovan LEM, Sartori IAM, Ribeiro Jr PD, Carvalho ACGS, Goiato MC. Evaluation of satisfaction of patients rehabilitated with zygomatic fixtures. J Oral Maxillofac Surg 2012; 70:314-319.

\section{AUTOR DE CORRESPONDENCIA:}

Prof. Orlando Guerra Cobián

Facultad de Estomatología

Avenida Carlos III y Avenida de los Presidentes.

Vedado

Ciudad de La Habana. Cuba

Tfno: 53-7-870 3312

Email: orlando.guerra@infomed.sld.cu 